\title{
Advanced High-Energy Resolution Silicon Drift Detectors
}

\author{
Ralf Terborg, Martin Rohde \\ Bruker AXS Microanalysis GmbH, Schwarzschildstr. 12, 12489 Berlin, Germany
}

Within the last decade, Silicon Drift Detectors (SDD) have become more and more popular in the field of X-ray detection and microanalysis. One of the significant differences in the functional principle to other solid state detectors has its origin in the SDD's design which is characterized by an additional electrical field parallel to the detector surface established by a set of additional contact strips, fig. 1a. The other important feature of these devices is the extremely low capacitance established by an integrated field effect transistor. The main characteristic of SDDs is their extremely high pulse load capacity of up to one million counts per second at good or reasonable energy resolution.

The third generation of SDD devices, the Silicon Drift Detector Droplet (SD ${ }^{3}$ ) with an optimized geometry [1], fig. 1b, combine the speed benefit with high quality light element performance and superior energy resolution values, even when compared with the best conventional $\mathrm{Si}(\mathrm{Li})$ detectors [2].

Besides other advances in detector technology like the development of a four-channel detector [3], further optimization now allows SDD systems with an energy resolution of around $125 \mathrm{eV}$ at $\mathrm{Mn}$ $\mathrm{K} \alpha$. These high performance SDD spectrometers show an improved energy resolution also in the low energy range, e.g. $59 \mathrm{eV}$ at F-K $\alpha$. This significantly improves the deconvolution accuracy of overlapping lines as well as quantitative light element analysis.

New signal electronic topologies, namely the pulsed charge reset principle, make it possible to maintain the excellent energy resolution values even at extreme count rates, fig.2, 3, thus rendering the inherent speed benefits of the SDD technology not only applicable for high speed mapping but also for high performance quantitative analysis. The latter enables new approaches in many fields such as defect analysis, mineralogy, or even gun shot residual measurements. An exemplary application is the discrimination of tungsten and silicon in a sample can be done by deconvolution and quantification of the W-M and Si-K series, fig. 4.

\section{References}

[1] Lechner et al., X-Ray Spectrom. 33, (2004), 256

[2] R. Terborg, M. Rohde, Microsc. Microanal. 9 (Suppl.2) Proceedings (2003), 120

[3] T. Schülein, R. Terborg. M. Rohde, Microsc. Microanal. 11 (Suppl.2) Proceedings (2005), 460 

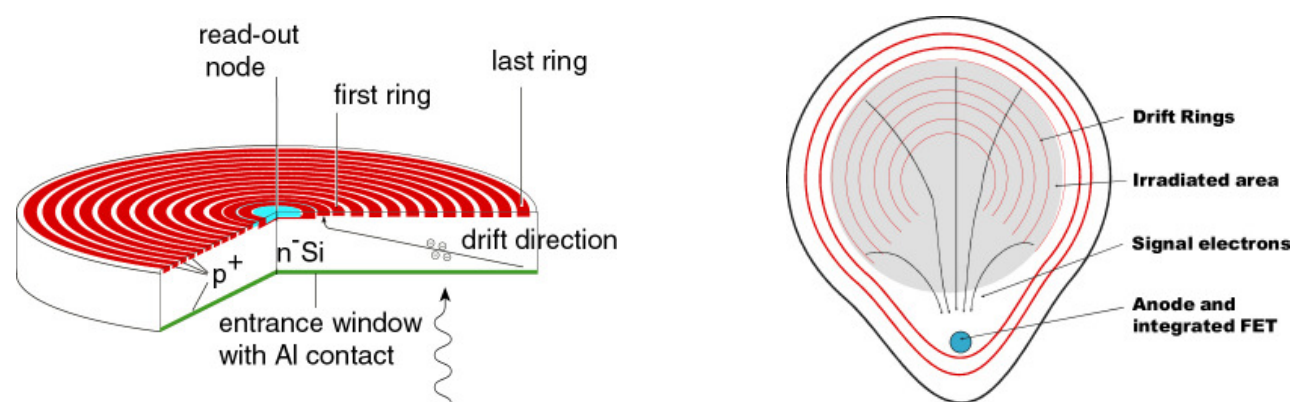

Fig. 1. Functional principle of (a) Silicon Drift Detector (SDD) and (b) Silicon Drift Detector Droplet (SD $\left.{ }^{3}\right)$

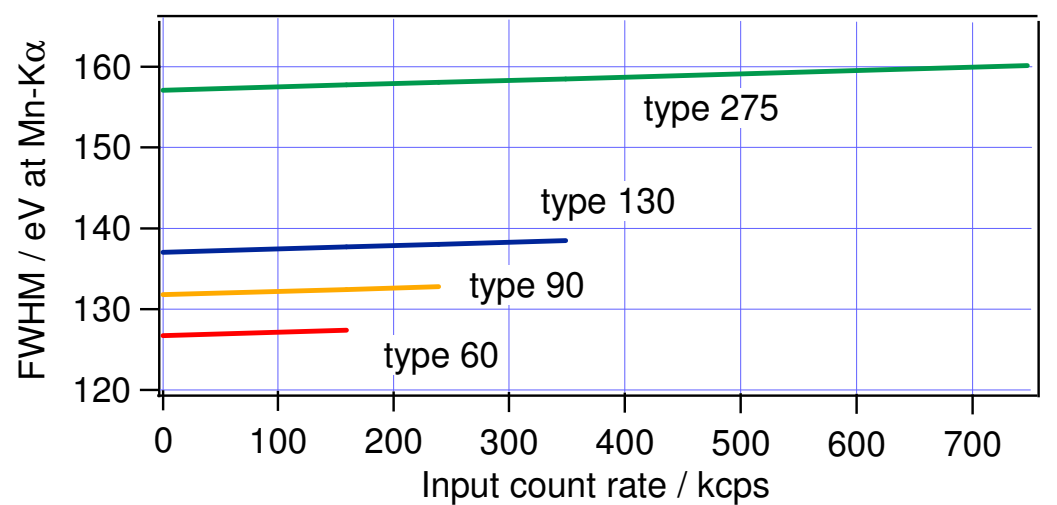

Fig. 2. Energy resolution (FWHM) of $\mathrm{SD}^{3}$ with different shaping amplifier types.

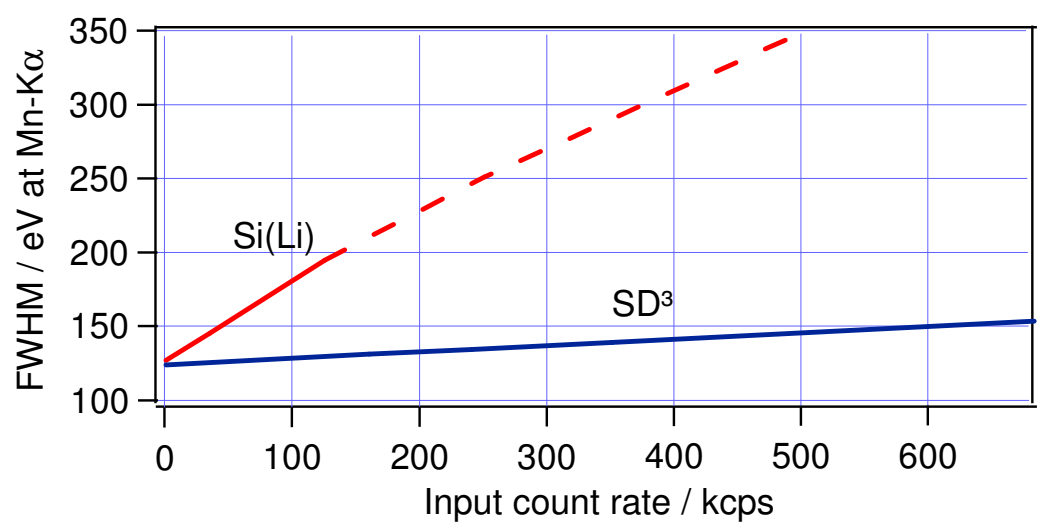

Fig. 3. Energy resolution (FWHM) of $\mathrm{SD}^{3}$ and state-of-the-art $\mathrm{Si}(\mathrm{Li})$ detectors at optimized shaping times.
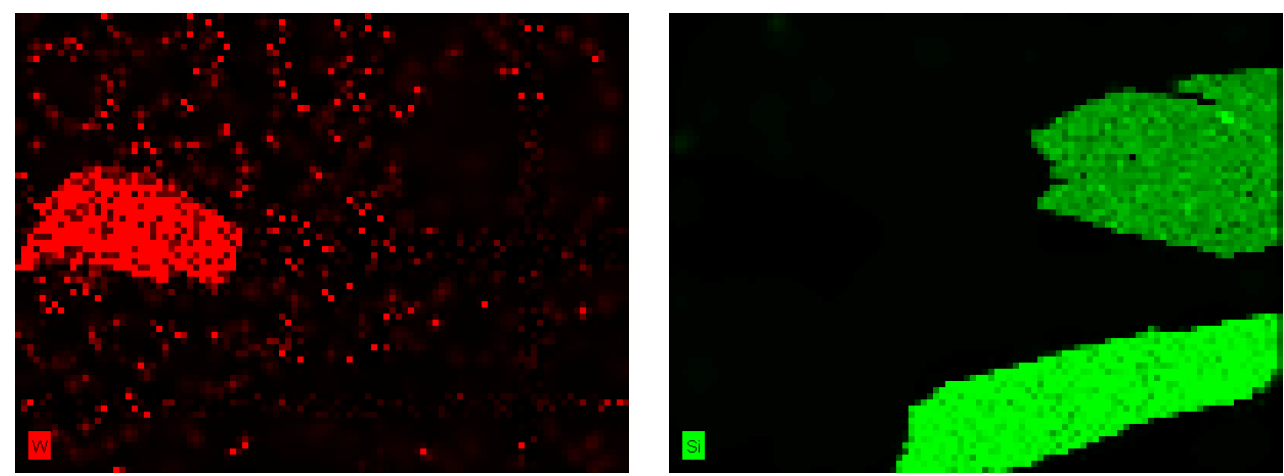

Fig. 4. Quantitative mapping of a $W$ and Si sample. Determination of the $W$ (left) and Si (right) components by discrimination of $\mathrm{Si}-\mathrm{K}$ and $\mathrm{W}$-M series. 